BMJ Open

Diabetes

Research

\& Care

\title{
Association of serum orosomucoid with 30-min plasma glucose and glucose excursion during oral glucose tolerance tests in non-obese young Japanese women
}

\author{
Ayaka Tsuboi, ${ }^{1,2}$ Satomi Minato,,${ }^{1,3}$ Megumu Yano, ${ }^{1}$ Mika Takeuchi, ${ }^{4}$ \\ Kaori Kitaoka, ${ }^{1,5}$ Miki Kurata, ${ }^{1,4}$ Gen Yoshino, ${ }^{6}$ Bin Wu, ${ }^{7,8}$ Tsutomu Kazumi, ${ }^{1,9}$ \\ Keisuke Fukuo ${ }^{1,4}$
}

To cite: Tsuboi A, Minato S, Yano M, et al. Association of serum orosomucoid with 30-min plasma glucose and glucose excursion during oral glucose tolerance tests in nonobese young Japanese women. BMJ Open Diab Res Care 2018;6:e000508. doi:10.1136/ bmjdrc-2018-000508

Received 2 January 2018 Revised 6 April 2018 Accepted 19 April 2018
Check for updates

For numbered affiliations see end of article.

Correspondence to Dr Tsutomu Kazumi; kazumi@mukogawa-u.ac.jp

\section{ABSTRACT}

Objective Inflammatory markers are elevated in insulin resistance (IR) and diabetes. We tested whether serum orosomucoid (ORM) is associated with postload glucose, $\beta$-cell dysfunction and IR inferred from plasma insulin kinetics during a $75 \mathrm{~g}$ oral glucose tolerance test (OGTT) Research design and methods $75 \mathrm{~g}$ 0GTTs were performed with multiple postload glucose and insulin measurements over a 30-120 min period in 168 nonobese Japanese women (aged 18-24 years). 0GTT responses, serum adiponectin and high-sensitivity $\mathrm{C}$ reactive protein (hsCRP) were cross-sectionally analyzed by analysis of variance and then Bonferroni's multiple comparison procedure. Stepwise multivariate linear regression analyses were used to identify most important determinants of ORM.

Results Of 168 women, 161 had normal glucose tolerance. Postload glucose levels and the area under the glucose curve $(\mathrm{AUCg})$ increased in a stepwise fashion from the first through the third ORM tertile. In contrast, there was no or modest, if any, association with fat mass index, trunk/leg fat ratio, adiponectin, hsCRP, postload insulinemia, the Matsuda index and homeostasis model assessment IR. In multivariable models, which incorporated the insulinogenic index, the Matsuda index and HOMA-IR, 30 min glucose (standardized $\beta$ : 0.517) and AUCg (standardized $\beta: 0.495$ ) explained $92.8 \%$ of ORM variations.

Conclusions Elevated circulating orosomucoid was associated with elevated 30 min glucose and glucose excursion in non-obese young Japanese women independently of adiposity, IR, insulin secretion, adiponectin and other investigated markers of inflammation. Although further research is needed, these results may suggest a clue to identify novel pathways that may have utility in monitoring dysglycemia within normal glucose tolerance.

\section{INTRODUCTION}

Subjects with impaired glucose tolerance (IGT) or impaired fasting glucose (IFG) have an increased risk for type 2 diabetes. ${ }^{1-3}$

\section{Significance of this study}

What is already known about this subject?

- Postload glucose and glucose excursion during an oral glucose tolerance test are related to insulin resistance and/or impaired insulin secretion, both of which are associated with obesity.

What are the new findings?

- Postload glucose and glucose excursion during an oral glucose tolerance test are related to serum orosomucoid in non-obese young Japanese women

- The relationships are independent of fat mass and distribution, markers of insulin resistance/ sensitivity, insulin secretion, adiponectin and other investigated markers of inflammation.

How might these results change the focus of research or clinical practice?

- Since insulin resistance and impaired insulin secretion are known to be causative in the development of diabetes, this might suggest that orosomucoid could be related to the glucose levels.

- These results may suggest a clue to identify novel pathways that may have utility in monitoring glucose dysmetabolism within normal glucose tolerance, although further research is needed.

Although more than half of the subjects who develop type 2 diabetes have IGT or IFG at baseline, ${ }^{4}$ prospective epidemiological studies have demonstrated that about $40 \%$ of subjects have normal glucose tolerance (NGT) at baseline. ${ }^{135}$ Therefore, other than fasting and 2-hour postload glucose, additional information might help us identify a group of subjects who might benefit from early lifestyle intervention.

Inflammation is recognized as a major etiological determinant of multiple disease 
states including cardiovascular disease, type 2 diabetes and metabolic syndrome. ${ }^{6}$ Several prospective studies have shown that high baseline levels of $\mathrm{C}$ reactive protein (CRP) are associated with the increased risk for type 2 diabetes. ${ }^{78}$ Among inflammatory markers, orosomucoid (ORM) has been less studied in relation to metabolic disorders, although the acute phase reactant protein is one of the most abundant plasma proteins, accounting for about $1 \%$ of all plasma proteins. ${ }^{9}$ In addition, most of the studies described above were conducted in Western countries where the prevalence of obesity is high. Recently, Qin et $a l^{10}$ have demonstrated that ORM activates AMP-activated protein kinase (AMPK) in skeletal muscle, which led to the activation of glycogen synthase and subsequent glycogen storage. As AMPK is important for glucose uptake and glycogen storage in the myocytes ${ }^{11}$ and skeletal muscle is responsible for the majority of glucose disposal, ${ }^{12}$ we aimed to examine whether ORM is associated with postchallenge glycemia in non-obese Japanese women and whether these associations, if any, depend on fat mass and distribution, insulin resistance (IR) and insulin secretion.

\section{METHODS}

We cross-sectionally studied 168 young women (50 collegiate athletes and 118 untrained students of the Mukogawa Women's University), aged 18-24 years whose details have been reported elsewhere. ${ }^{13}{ }^{14}$ Athletes were students of the Department of Health and Sports Sciences and were also members of volleyball club, basketball club or track club (middle-distance runners) of the University. They had been training regularly for 2 years or longer prior to the study, 5 hours a day and 6 days a week and participated regularly in competitive events in their respective sport specialties. Non-athletes were students of the Department of Food Sciences and Nutrition. Although they were not engaged in any regular sport activity, they had $9367 \pm 1971$ steps/day (mean \pm SD of 77 non-athletes, who used a pedometer for 14 days and mean steps a day were calculated in each participants). This may explain in part the result that the difference in insulin sensitivity was small between athletes and non-athletes as previously reported. ${ }^{13}$ Subjects with clinically diagnosed acute or chronic inflammatory diseases, endocrine, cardiovascular, hepatic, renal diseases, hormonal contraception and unusual dietary habits were excluded. Nobody reported to receive any medications or have regular supplements. The study was in accordance with the Helsinki Declaration. All subjects were recruited as volunteers and gave written consent after the experimental procedure had been explained.

After a 12 hours overnight fast, a standard $75 \mathrm{~g}$ OGTT with glucose and insulin measurements at 0 (fasting), $30 \mathrm{~min}, 1$ hour and 2 hours was performed in the morning. Plasma glucose was determined by the hexokinase/ glucose-6-phosphate dehydrogenase method (interassay coefficient of variation (CV) $<2 \%$ ). Serum insulin was measured by an ELISA method with a narrow specificity excluding des-31, des-32 and intact proinsulin (interassay $\mathrm{CV}<6 \%$ ). Area under the response curve of plasma glucose (AUCg) and serum insulin (AUCi) was calculated by the trapezoidal method. Homeostasis model assessment-IR (HOMA-IR), the Matsuda index and the insulinogenic index (IGI) were calculated as previously reported. ${ }^{15-17}$

Leptin, adiponectin, high-sensitivity CRP (hsCRP) and plasminogen activator inhibitor-1 (PAI-1) were measured in fasted samples as previously reported. ${ }^{13} 14$ ORM concentrations were measured in fasted samples by an immunoturbidimetric method using a commercially available kit (N Antiserum to Human $\alpha 1$-acid Glycoprotein, Siemens Healthcare Diagnostics, Tokyo, Japan) and an autoanalyzer (JCA-BM6010, JEOL, Tokyo, Japan). Intra-assay and interassay $\mathrm{CV}$ at $87 \mathrm{mg} / \mathrm{dL}$ was $1.4 \%$ and $1.7 \%$, respectively.

Fat mass, bone mass and lean mass for arms, legs, trunk and the total body were measured using whole-body dual-energy X-ray absorptiometry (Hologic QDR-2000, software V.7.20D, Waltham, Massachusetts, USA) as previously reported. ${ }^{13} 14$ The leg region included the entire hip, thigh and leg. General adiposity was assessed using height-adjusted and weight-adjusted body fat. The former, fat mass index (FMI), was calculated as body fat mass in $\mathrm{kg}$ divided by height in meter squared and the latter, per cent body fat, was calculated as body fat mass in $\mathrm{kg}$ divided by body weight in $\mathrm{kg} \times 100$. Abdominal fat accumulation was assessed by the ratio of trunk fat-to-leg fat. ${ }^{18}$

Data were presented as mean $\pm \mathrm{SD}$ unless otherwise stated. Due to deviation from normal distribution, IGI and hsCRP were logarithmically transformed for analyses. Cross-sectional differences were tested using analysis of variance among ORM tertile and then Bonferroni's multiple comparison procedure. Associations of ORM were analyzed by Pearson's correlation. In order to identify most important determinants of ORM, stepwise multivariate linear regression analyses were performed. Independent variables included were all variables that showed significant difference among ORM tertile. Comparisons of ORM levels between athletes and non-athletes were made with t-test. A two-tailed value of $\mathrm{p}<0.05$ was considered significant. Statistics were performed with SPSS system V.17.0 (SPSS, Chicago, Illinois, USA).

\section{RESULTS}

Of 168 women studied, 2 athletes and 1 non-athlete had IFG, 4 non-athletes had IGT and 161 had NGT. Because serum ORM levels did not differ between athletes and non-athletes $(126 \pm 28$ vs $127 \pm 26 \mathrm{mg} / \mathrm{dL}, \mathrm{p}=0.87)$, both groups were combined to be analyzed to obtain wider range of adiposity. Table 1 displays characteristics of women grouped by ORM tertile $(<112,113-134$, $\geq 135 \mu \mathrm{g} / \mathrm{mL}$ ). As previously reported, ${ }^{13} 14$ young women were normal weight, had normal waist circumference 
Table 1 Features of 168 young Japanese women grouped by tertile of serum orosomucoid concentrations

\begin{tabular}{|c|c|c|c|c|}
\hline Tertile & First & Second & Third & \\
\hline Range (mg/dL) & $<112$ & $113-134$ & $\geq 135$ & \\
\hline Orosomucoid (mg/dL) & $100 \pm 10$ & $125 \pm 6$ & $155 \pm 20$ & $a b c$ \\
\hline Age (years) & $20.3 \pm 1.1$ & $20.3 \pm 1.0$ & $20.6 \pm 1.1$ & \\
\hline Fat mass index $\left(\mathrm{kg} / \mathrm{m}^{2}\right)$ & $5.6 \pm 1.6$ & $5.3 \pm 1.8$ & $5.2 \pm 1.8$ & \\
\hline Waist circumference $(\mathrm{cm})$ & $73.3 \pm 5.5$ & $73.5 \pm 5.5$ & $72.9 \pm 5.9$ & \\
\hline Percentage body fat (\%) & $26.6 \pm 6.1$ & $25.5 \pm 6.1$ & $25.2 \pm 6.3$ & \\
\hline Trunk/leg fat ratio & $1.24 \pm 0.24$ & $1.24 \pm 0.25$ & $1.21 \pm 0.24$ & \\
\hline Leptin (ng/mL) & $7.7 \pm 3.5$ & $7.3 \pm 3.5$ & $7.6 \pm 4.2$ & \\
\hline Adiponectin $(\mu \mathrm{g} / \mathrm{mL})$ & $13.4 \pm 5.1$ & $12.2 \pm 4.5$ & $11.6 \pm 4.1$ & \\
\hline $\mathrm{hsCRP}(\mu \mathrm{g} / \mathrm{dL})$ & $44 \pm 97$ & $23 \pm 33$ & $32 \pm 48$ & \\
\hline PAl-1 (ng/mL) & $24 \pm 9$ & $29 \pm 14$ & $26 \pm 13$ & \\
\hline Fasting glucose (mg/dL) & $82 \pm 6$ & $84 \pm 6$ & $86 \pm 7$ & $\mathrm{~b}$ \\
\hline $30 \mathrm{~min}$ glucose $(\mathrm{mg} / \mathrm{dL})$ & $97 \pm 13$ & $123 \pm 8$ & $148 \pm 20$ & $a b c$ \\
\hline 1 hour glucose (mg/dL) & $79 \pm 17$ & $100 \pm 18$ & $129 \pm 35$ & $a b c$ \\
\hline 2 hours glucose $(\mathrm{mg} / \mathrm{dL})$ & $80 \pm 12$ & $91 \pm 17$ & $104 \pm 24$ & $a b c$ \\
\hline Fasting insulin $(\mu \mathrm{U} / \mathrm{mL})$ & $5.8 \pm 3.5$ & $5.7 \pm 2.3$ & $5.9 \pm 3.4$ & \\
\hline 30 min insulin $(\mu \mathrm{U} / \mathrm{mL})$ & $56 \pm 35$ & $53 \pm 25$ & $52 \pm 33$ & \\
\hline 1 hour insulin $(\mu \mathrm{U} / \mathrm{mL})$ & $32 \pm 17$ & $43 \pm 20$ & $52 \pm 31$ & $a b$ \\
\hline 2 hours insulin $(\mu \mathrm{U} / \mathrm{mL})$ & $32 \pm 25$ & $35 \pm 20$ & $42 \pm 25$ & \\
\hline$\Delta$ glucoe30 (mg/dL) & $15 \pm 14$ & $40 \pm 8$ & $62 \pm 18$ & $a b c$ \\
\hline$\Delta$ insulin30 $(\mu \mathrm{U} / \mathrm{mL})$ & $50 \pm 35$ & $47 \pm 24$ & $46 \pm 32$ & \\
\hline AUCg (mg/dL, 2hours) & $168 \pm 18$ & $203 \pm 19$ & $245 \pm 38$ & $a b c$ \\
\hline AUCi $(\mu \mathrm{U} / \mathrm{mL}, 2$ hours $)$ & $70 \pm 32$ & $78 \pm 29$ & $87 \pm 43$ & $\mathrm{~b}$ \\
\hline HOMA-IR & $1.17 \pm 0.69$ & $1.19 \pm 0.51$ & $1.27 \pm 0.80$ & \\
\hline Matsuda index & $10.8 \pm 4.2$ & $9.1 \pm 3.6$ & $8.8 \pm 4.7$ & $\mathrm{~b}$ \\
\hline IGI & $10.2 \pm 20.9$ & $1.2 \pm 0.6$ & $1.2 \pm 3.4$ & $a b$ \\
\hline Athletes (n, \%) & 1730.4 & $18,32.1$ & $15,26.8$ & \\
\hline
\end{tabular}

Mean $\pm S D(n=56)$ in each tertile. Significantly different at $p<0.05$ or less.

$\mathrm{a}$ and $\mathrm{b}$ : first vs second and third tertile, respectively, c: second vs third tertile.

AUCg, area under the concentration curve of glucose; AUCi, area under the concentration curve of insulin; FMI, fat mass index; $\Delta$ glucose30 and $\Delta$ insulin30, incremental $30 \mathrm{~min}$ glucose and insulin concentrations, respectively; HOMA-IR, homeostasis model assessment of insulin resistance; hsCRP, high-sensitivity C reactive protein; IGI, insulinogenic index; PAI-1, plasminogen activator inhibitor-1.

and hence were insulin sensitive as indicated by normal fasting insulin levels.

ORM levels were not associated with FMI, the percentage of body fat, trunk/leg fat ratio and serum leptin (table 1). In addition, ORM was not associated with serum hsCRP and PAI-1. Furthermore, no difference was found in serum adiponectin among three groups as well. In contrast, as ORM increased from the first through the third tertile (figure 1), postload glucose levels at 3 points of time increased in a stepwise fashion in both non-athletes and athletes, whereas postload insulin levels did not differ except for 1 hour insulin (table 1). AUCg and an incremental 30 min glucose concentration increased in a stepwise fashion, whereas an incremental $30 \mathrm{~min}$ insulin concentration did not differ (table 1). Women with the third (higher ORM) as compared with the first ORM tertile had lower IGI and the Matsuda index, and higher fasting glucose, 1 hour insulin and AUCi. HOMA-IR and the percentage of athletes did not differ.

Multivariate stepwise linear regression analyses for ORM as a dependent variable was done, which included postload glucose values at 3 points of time, AUCg, IGI ( $\log$ transformed), the Matsuda index and HOMA-IR as independent variables (table 2). AUCg and $30 \mathrm{~min}$ glucose were independent determinants of ORM and explained $92.8 \%$ of variations of ORM. Subgroup analyses in athletes and non-athletes revealed similar results (table 2). Figure 2 shows the scatter plot between serum ORM and 30 min glucose and AUCg.

\section{DISCUSSION}

The present study has demonstrated that increased ORM was associated with increased glucose excursion 

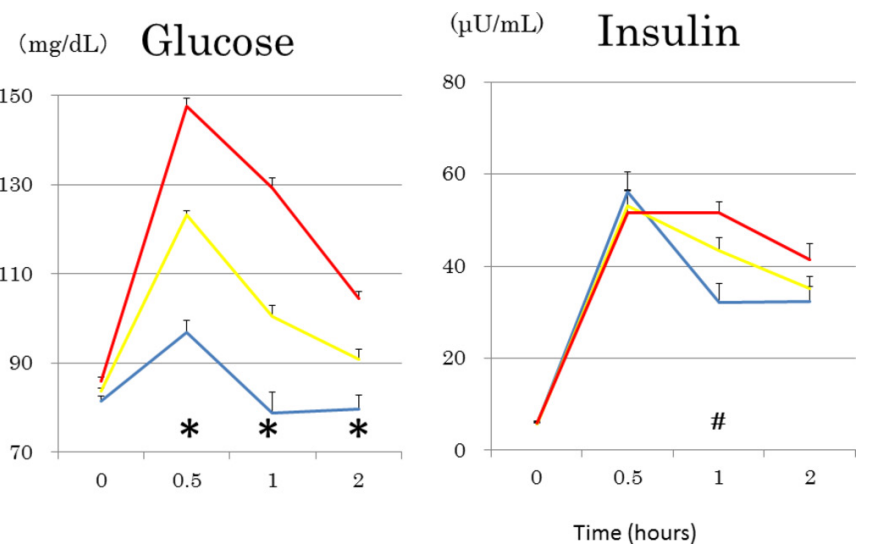

Figure 1 Mean \pm SEs of glucose curves during $75 \mathrm{~g}$ oral glucose testing in young Japanese women divided into three groups according to tertile of serum orosomucoid: low, blue; median, yellow; high, red. *Under blue lines; glucose values at this time points are significantly different from each other among three tertile groups at $p<0.05$ or less. "Insulin values at 1 hour were significantly lower in the low as compared with the median and the high orosomucoid tertile. $n=56$ in each tertile. Range of orosomucoid (in mg/dL); low: $<112$, median: 113-134, high: $\geq 135$.

(AUCg) and $30 \mathrm{~min}$ postload glucose in non-obese, young Japanese women. These associations were not related to sophisticated measures of general and abdominal adiposity (FMI, the percentage of body fat, trunk/ leg fat ratio) and serum leptin. Associations observed were not related to insulin secretion, insulin sensitivity/ resistance, adiponectin and other investigated markers of inflammation. Glucose excursion (AUCg) and $30 \mathrm{~min}$ postload glucose explained $>90 \%$ of variations of ORM. The present results in young women confirmed positive associations of ORM with fasting and 2-hour glucose as previously reported in general population, and in overweight and obese people. ${ }^{19-21}$

Metabolomics now seeks to identify early biomarkers which are predictive of the development of dysglycemia and type 2 diabetes. A Finnish metabolic profiling study ${ }^{22}$ demonstrated that branched-chain and aromatic amino acids and orosomucoid referred to as $\alpha 1$-acid glycoprotein, were predictors of both fasting and 2-hour glucose
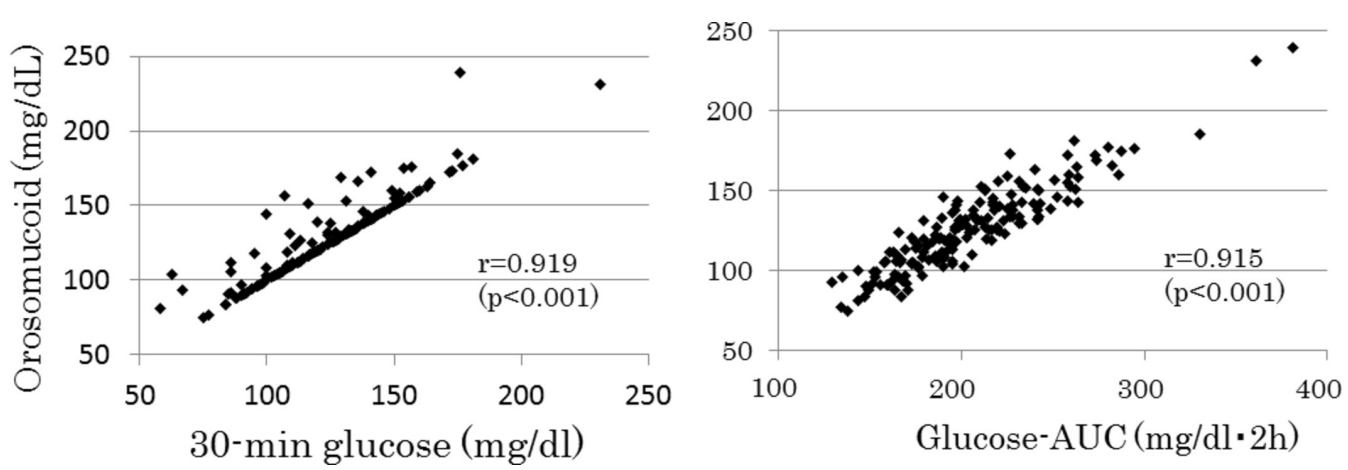

Figure 2 The scatter plot between serum orosomucoid and $30 \mathrm{~min}$ glucose and the area under the glucose curve (glucoseAUC) during a $75 \mathrm{~g}$ oral glucose tolerance test.

Table 2 Multivariate stepwise linear regression analysis for orosomucoid as a dependent variable

\begin{tabular}{llll}
\hline $\begin{array}{l}\text { Non- } \\
\text { athletes }\end{array}$ & Standardized $\boldsymbol{\beta}$ & Cumulative $\mathbf{R}^{2}$ & $\mathbf{P}$ values \\
\hline AUCg & 0.565 & 0.837 & $2.49 \mathrm{E}-22$ \\
$\begin{array}{l}30 \mathrm{~min} \\
\text { glucose }\end{array}$ & 0.440 & 0.907 & $4.86 \mathrm{E}-16$ \\
\hline $\begin{array}{l}\text { Athletes } \\
\quad \begin{array}{l}30 \mathrm{~min} \\
\text { glucose }\end{array}\end{array}$ & 0.781 & 0.969 & $1.86 \mathrm{E}-20$ \\
$\begin{array}{l}\text { AUCg } \\
\text { Total }\end{array}$ & 0.225 & 0.978 & $3.56 \mathrm{E}-05$ \\
\hline $\begin{array}{l}30 \mathrm{~min} \\
\text { glucose }\end{array}$ & 0.517 & & \\
\hline AUCg & 0.495 & 0.844 & $3.39 \mathrm{E}-31$ \\
\hline
\end{tabular}

Independent variables included are the same in all models: postload glucose levels at 3 points of time, AUCg, log IGI, HOMAIR and Matsuda index.

AUCg, area under the concentration curve of glucose; HOMAIR, homeostasis model assessment of insulin resistance; IGI, insulinogenic index.

at 6.5-year follow-up in a general population setting of middle-aged men and women.

Since the prevailing insulin concentrations over the first $30 \mathrm{~min}$ of the OGTT, a direct estimate of insulin secretion during the OGTT, were not associated with ORM, insufficient insulin secretion was possibly not the primary factor. Studies from Asia have reported an independent association between elevated $30 \mathrm{~min}$ glucose and incident diabetes. ${ }^{23}{ }^{24}$ However, this was associated with decreased $\beta$-cell function in those studies. ${ }^{23}{ }^{24} \mathrm{An}$ incremental $30 \mathrm{~min}$ glucose concentration may reflect the ability of endogenous insulin secretion to suppress hepatic glucose production. ${ }^{25}$ It is suggested that hepatic IR during early phase insulin secretion may be related to early phase hyperglycemia. ${ }^{25}$ Although there was no association between ORM and HOMA-IR, a more reflective surrogate of hepatic IR, ${ }^{16}$ the possibility cannot be ruled out that hepatic IR may be involved in ORM-early phase 
hyperglycemia association because majority of circulating ORM is produced in the liver. ${ }^{21}$

In above-mentioned studies, ${ }^{19-22}$ serum ORM concentrations were considered a marker of low-grade chronic inflammation, which links obesity to IR and $\beta$-cell failure resulting in dysglycemia. ${ }^{26}$ However, in the present study, there was no association of ORM with FMI, trunk/leg fat ratio, hsCRP and adiponectin, the last of which is known to stimulate glucose uptake through AMPK or peroxisome proliferator-activated receptor $\alpha$ activation. ${ }^{27}$ Since IR and $\beta$-cell failure are known to be causative in the development of diabetes, this might suggest that ORM could be related to the glucose levels independently of the muscle insulin signaling: insulin-independent glucose uptake via skeletal muscle.

It is reported that contraction-stimulated, insulin-independent glucose transport in skeletal muscle may be mediated by AMPK. ${ }^{28}{ }^{29}$ Studies in mice have demonstrated that contraction-stimulated elevation of muscle ORM increases muscle glycogen and enhances muscle endurance, ${ }^{30}$ which is mediated through activation of AMPK by ORM in skeletal muscle. ${ }^{10}$ Taken together, it seems reasonable to speculate that ORM per se may stimulate glucose uptake by myocytes and hence may lower blood glucose. Indeed, exogenous ORM improved glucose and insulin tolerance in diabetic $\mathrm{db} / \mathrm{db}$ mice. ${ }^{21}$ However, in the present study, higher serum ORM was associated with higher, but not lower glycemia. It may be that sustained increase in circulating ORM might cause ORM resistance. ${ }^{21}$

Increased ORM may be a consequence of increased plasma glucose in the present study as it is reported that adipose ORM levels were elevated by high glucose ${ }^{31}$ and it has been suggested to modulate immune responses to protect adipose tissue from inflammation and metabolic dysfunction. ${ }^{21}$ However, this explanation appears to be unlikely in the present study because differentiated adipocytes were incubated with extremely high glucose $(450 \mathrm{mg} / \mathrm{dL})^{31}$ and because another study reported repressed adipose ORM expression in diabetic ob/ob mice, although ORM was expressed in adipose tissue at high levels. ${ }^{32}$ The biology underlying the association between high serum ORM concentrations and increased glucose excursion found in the present study is not known.

The strengths of the present study include homogeneous study population with scarce confounding factors, and accurate and reliable measures of body composition by dual-energy X-ray absorptiometry (DXA). Several limitations of this study warrant consideration. The cross-sectional design of the present study complicates the drawing of causal inferences, and a single measurement of biochemical variables may be susceptible to short-term variation, which would bias the results towards the null. We used crude measures of insulin sensitivity/IR and insulin secretion, which may be less accurate. Statistical power was not calculated. As we studied young Japanese women only, results may not be generalized to other gender, age populations, races or ethnicities.

In conclusion, elevated circulating orosomucoid was associated with elevated $30 \mathrm{~min}$ glucose and glucose excursion in non-obese young Japanese women independently of general and abdominal adiposity, insulin secretion, insulin sensitivity/resistance, other investigated markers of inflammation and adiponectin. Further research is needed to determine the public health and clinical significance of these results. But these results may suggest a clue to identify novel pathways that may have utility in monitoring glucose dysmetabolism within NGT.

\section{Author affiliations}

${ }^{1}$ Research Institute for Nutrition Sciences, Mukogawa Women's University, Nishinomiya, Japan

${ }^{2}$ Department of Nutrition, Osaka City Juso Hospital, Osaka, Japan

${ }^{3}$ Graduate School of Human Science and Environment, University of Hyogo, Himeji, Japan

${ }^{4}$ Department of Food Sciences and Nutrition, School of Human Environmental Sciences, Mukogawa Women's University, Nishinomiya, Japan

${ }^{5}$ Department of Nutritional Sciences for Well-being, Faculty of Health Sciences for Welfare, Kansai University of Welfare Sciences, Kashiwara, Japan

${ }^{6}$ Diabetes Center, Shinsuma Hospital, Kobe, Japan

${ }^{7}$ Open Research Center for Studying of Lifestyle-Related Diseases, Mukogawa Women's University, Nishinomiya, Japan

${ }^{8}$ Department of Endocrinology, First Affiliated Hospital of Kunming Medical University, Kunming, China

${ }^{9}$ Department of Medicine, Kohnan Kakogawa Hospital, Kakogawa, Japan

Acknowledgements The authors would like to thank all participants for their dedicated and conscientious collaboration.

Contributors AT, SM, MY, MT, KK, MK, GY and BW collected and analyzed data. TK wrote the manuscript and KF reviewed and edited it. All authors approved the final version of the manuscript to be published. TK supervised the study, had full access to all the data in the study and takes responsibility for the integrity of the data and the accuracy of the data analysis.

Funding This research received no specific grant from any funding agency in the public, commercial or not-for-profit sectors.

Competing interests None declared.

Patient consent Obtained.

Ethics approval The Ethics Committees of the Mukogawa Women's University (No. 07-28).

Provenance and peer review Not commissioned; externally peer reviewed. Data sharing statement The ethics committees of the University does not allow us to open data except for a manuscript.

Open Access This is an Open Access article distributed in accordance with the Creative Commons Attribution Non Commercial (CC BY-NC 4.0) license, which permits others to distribute, remix, adapt, build upon this work non-commercially, and license their derivative works on different terms, provided the original work is properly cited and the use is non-commercial. See: http://creativecommons.org/ licenses/by-nc/4.0/

(C) Article author(s) (or their employer(s) unless otherwise stated in the text of the article) 2018. All rights reserved. No commercial use is permitted unless otherwise expressly granted.

\section{REFERENCES}

1. Gerstein HC, Santaguida P, Raina P, et al. Annual incidence and relative risk of diabetes in people with various categories of dysglycemia: a systematic overview and meta-analysis of prospective studies. Diabetes Res Clin Pract 2007;78:305-12. 
2. Shaw JE, Zimmet PZ, de Courten M, et al. Impaired fasting glucose or impaired glucose tolerance. What best predicts future diabetes in Mauritius? Diabetes Care 1999;22:399-402.

3. Gabir MM, Hanson RL, Dabelea D, et al. The 1997 American Diabetes Association and 1999 World Health Organization criteria for hyperglycemia in the diagnosis and prediction of diabetes. Diabetes Care 2000;23:1108-12.

4. Unwin N, Shaw J, Zimmet P, et al. Impaired glucose tolerance and impaired fasting glycaemia: the current status on definition and intervention. Diabet Med 2002;19:708-23.

5. Eschwège $\mathrm{E}$, Charles MA, Simon D, et al. Reproducibility of the diagnosis of diabetes over a 30-month follow-up: the Paris Prospective Study. Diabetes Care 2001;24:1941-4.

6. Ridker PM. Inflammatory biomarkers and risks of myocardial infarction, stroke, diabetes, and total mortality: implications for longevity. Nutr Rev 2007;65(12 Pt 2):253-9.

7. Hu FB, Meigs JB, Li TY, et al. Inflammatory markers and risk of developing type 2 diabetes in women. Diabetes 2004;53:693-700.

8. Wang X, Bao W, Liu J, et al. Inflammatory markers and risk of type 2 diabetes: a systematic review and meta-analysis. Diabetes Care 2013;36:166-75.

9. Fournier T, Medjoubi-N N, Porquet D. Alpha-1-acid glycoprotein. Biochim Biophys Acta 2000;1482(1-2):157-71.

10. Qin Z, Wan JJ, Sun Y, et al. ORM promotes skeletal muscle glycogen accumulation via CCR5-activated AMPK Pathway in mice. Front Pharmacol 2016;7:302.

11. Steinberg GR, Kemp BE. AMPK in Health and Disease. Physiol Rev 2009;89:1025-78.

12. Bonadonna RC, Saccomani MP, Seely L, et al. Glucose transport in human skeletal muscle. The in vivo response to insulin. Diabetes 1993;42:191-8.

13. Kitaoka K, Takeuchi M, Tsuboi A, et al. Increased adipose and muscle insulin sensitivity without changes in serum adiponectin in young female collegiate athletes. Metab Syndr Relat Disord 2017;15:246-51.

14. Tanaka S, Wu B, Honda M, et al. Associations of lower-body fat mass with favorable profile of lipoproteins and adipokines in healthy, slim women in early adulthood. J Atheroscler Thromb 2011;18:365-72.

15. Stumvoll M, Mitrakou A, Pimenta W, et al. Use of the oral glucose tolerance test to assess insulin release and insulin sensitivity. Diabetes Care 2000;23:295-301.

16. Matthews DR, Hosker JP, Rudenski AS, et al. Homeostasis model assessment: insulin resistance and beta-cell function from fasting plasma glucose and insulin concentrations in man. Diabetologia 1985;28:412-9.

17. Matsuda M, DeFronzo RA. Insulin sensitivity indices obtained from oral glucose tolerance testing: comparison with the euglycemic insulin clamp. Diabetes Care 1999;22:1462-70.
18. Lim U, Turner SD, Franke AA, et al. Predicting total, abdominal, visceral and hepatic adiposity with circulating biomarkers in Caucasian and Japanese American women. PLoS One 2012; 7:e43502.

19. Schmidt MI, Duncan BB, Sharrett AR, et al. Markers of inflammation and prediction of diabetes mellitus in adults (Atherosclerosis Risk in Communities study): a cohort study. Lancet 1999;353:1649-52.

20. Alfadda AA, Fatma S, Chishti MA, et al. Orosomucoid serum concentrations and fat depot-specific mRNA and protein expression in humans. Mol Cells 2012:33:35-41.

21. Lee YS, Choi JW, Hwang I, et al. Adipocytokine orosomucoid integrates inflammatory and metabolic signals to preserve energy homeostasis by resolving immoderate inflammation. $\mathrm{J} \mathrm{Biol} \mathrm{Chem}$ 2010;285:22174-85.

22. Würtz $P$, Tiainen $M$, Mäkinen VP, et al. Circulating metabolite predictors of glycemia in middle-aged men and women. Diabetes Care 2012;35:1749-56.

23. Chamukuttan S, Ram J, Nanditha A, et al. Baseline level of 30-min plasma glucose is an independent predictor of incident diabetes among Asian Indians: analysis of two diabetes prevention programmes. Diabetes Metab Res Rev 2016;32:762-7.

24. Yang HK, Ha HS, Rhee M, et al. Predictive value of glucose parameters obtained from oral glucose tolerance tests in identifying individuals at high risk for the development of diabetes in Korean Population. Medicine 2016;95:e3053.

25. DiPietro L, Dziura J, Yeckel CW. Specific relation between abdominal obesity and early-phase hyperglycemia is modulated by hepatic insulin resistance in healthy older women. Diabetes Care 2010;33:165-7.

26. Khodabandehloo H, Gorgani-Firuzjaee S, Panahi G, et al. Molecular and cellular mechanisms linking inflammation to insulin resistance and $\beta$-cell dysfunction. Transl Res 2016;167:228-56.

27. Kadowaki T, Yamauchi T. Adiponectin and adiponectin receptors. Endocr Rev 2005;26:439-51.

28. Hayashi T, Hirshman MF, Kurth EJ, et al. Evidence for 5' AMPactivated protein kinase mediation of the effect of muscle contraction on glucose transport. Diabetes 1998;47:1369-73.

29. Hunter RW, Treebak JT, Wojtaszewski JF, et al. Molecular mechanism by which AMP-activated protein kinase activation promotes glycogen accumulation in muscle. Diabetes 2011;60:766-74.

30. Lei H, Sun Y, Luo Z, et al. Fatigue-induced Orosomucoid 1 Acts on C-C Chemokine Receptor Type 5 to Enhance Muscle Endurance. Sci Rep 2016;6:18839.

31. Lin $\mathrm{Y}$, Berg $A H$, lyengar $P$, et al. The hyperglycemia-induced inflammatory response in adipocytes: the role of reactive oxygen species. J Biol Chem 2005;280:4617-26.

32. Lin Y, Rajala MW, Berger JP, et al. Hyperglycemia-induced production of acute phase reactants in adipose tissue. J Biol Chem 2001;276:42077-83. 